\title{
Coupled-cluster single-double calculations of the relativistic energy shifts in C IV, Na I, Mg II, Al III, Si IV, Ca II and Zn II
}

\author{
V. A. Dzuba* \\ School of Physics, University of New South Wales, Sydney 2052, Australia \\ W. R. Johnson话 \\ Department of Physics, 225 Nieuwland Science Hall \\ University of Notre Dame, Notre Dame, IN 46566
}

(Dated: September 15, 2021)

\begin{abstract}
The relativistic coupled-cluster single-double method is used to calculate the dependence of frequencies of strong E1-transitions in many monovalent atoms and ions on the fine-structure constant $\alpha$. These transitions are used in the search for manifestations of the variation of the fine-structure constant in quasar absorption spectra. Results of the present calculations are in good agreement with previous calculations but are more accurate.
\end{abstract}

PACS numbers: PACS: 31.30.Jv, 06.20.Jr,95.30.Dr

\section{INTRODUCTION}

Theories unifying gravity with other interactions suggest a possibility of temporal and spatial variations of the fundamental constants of nature; a review of these theories and results of measurement can be found in Ref. [1]. A very sensitive many-multiplet (MM) method to search for the variation of the fine-structure constant $\alpha=e^{2} / \hbar c$ by comparison of quasar absorption spectra with laboratory spectra has been suggested in Refs. [2, 3].

Using this method, strong evidence that the finestructure constant might be smaller about ten billion years ago was found [2, 4, 5, 6, 7, 8]. This result was obtained from an analysis of data from the Keck telescope in Hawaii by the group based at the University of New South Wales in Australia. However, an analysis of data from the VLT telescope in Chile, performed by different groups [9, 10] using the same MM method, gave a null result. There is an outgoing debate in the literature about possible reasons for the disagreement.

The MM method requires calculation of relativistic corrections to frequencies of atomic transitions to reveal their dependence on the fine-structure constant. All calculations used in the analysis so far were performed by a single research group based at the University of New South Wales 14, 15, 16, 17, 18, 19, 20]. Owing to the importance of detecting variations of fundamental constants and the disagreement mentioned above, it is important that the related atomic calculations be verified independently.

A positive development in this direction is a recent independent calculation of the relativistic energy shifts in the ion Fe II 21]. Fe II is the single most important element in the analysis of quasar absorption spectra. It has lines which move in opposite directions when $\alpha$ varies;

\footnotetext{
*Electronic address: V.Dzuba@unsw.edu.au

${ }^{\dagger}$ Electronic address: johnson@nd.edu URL: www.nd.edu/ johnson
}

moreover, the shifts in Fe II are relatively large. In principle, subject to sufficient statistics, Fe II alone could serve as a probe of variation of the fine-structure constant in quasar absorption spectra 21]. However, calculations for Fe II are difficult due to the large number of valence electrons.

In a recent work 22] independent calculations of the relativistic energy shifts for monovalent and diavalent atoms of astrophysical interest were reported. This work also presented a detailed analysis of Breit contributions to the relativistic energy shift. Note that, despite some overlap of authors of early [14, 15, 16, 17, 18, 19, 20] and recent [21, 22] works, the later can be regarded as independent since they use completely independent sets of computer codes and different methods of calculation.

In present work, we study the role of higher-order correlations in relativistic energy shifts of monovalent atoms and ions of astrophysical interest. We use a linearized coupled-cluster method in the single-double approximation and third-order many-body perturbation theory to perform the calculations. We demonstrate that including higher-order correlations significantly improves the accuracy of the transition energies. The values of relativistic energy shifts found in the present work are in good agreement with previous calculations but are more accurate. Note also that the present work can be considered as an independent verification of earlier calculations. This is because the calculations are performed using a method that has never before been used for this purpose.

Apart from the important task of calculating relativistic energy shifts of atomic frequencies of astrophysical interest, this work can be considered as another demonstration of the power of the single-double method. It applies the method for the first time to the ions C IV and Zn II. 


\section{METHOD}

It is convenient to present the dependence of atomic frequencies on the fine-structure constant $\alpha$ in the vicinity of its physical value $\alpha_{0}$ in the form

$$
\omega(x)=\omega_{0}+q x,
$$

where $\omega_{0}$ is the laboratory value of the frequency and $x=\left(\alpha / \alpha_{0}\right)^{2}-1, q$ is the coefficient which is to be found from atomic calculations. Note that

$$
q=\left.\frac{d \omega}{d x}\right|_{x=0} .
$$

To calculate this derivative numerically we use

$$
q \approx \frac{\omega(+\delta)-\omega(-\delta)}{2 \delta}
$$

Here $\delta$ must be small to exclude non-linear in $\alpha^{2}$ terms. In the present calculations we use $\delta=0.05$, which leads to

$$
q \approx 10(\omega(+0.05)-\omega(-0.05)) .
$$

To calculate the coefficients $q$ using (3), $\alpha$ must be varied in our computer codes. Therefore, it is convenient to use a form of the single electron wave function in which the dependence on $\alpha$ is explicitly shown (we use atomic units in which $e=\hbar=1, \alpha=1 / c$ )

$$
\psi(r)_{n j l m}=\frac{1}{r}\left(\begin{array}{c}
f_{v}(r) \Omega(\mathbf{n})_{j l m} \\
i \alpha g_{v}(r) \widetilde{\Omega}(\mathbf{n})_{j l m}
\end{array}\right),
$$

where $n$ is the principal quantum number and an index $v$ replaces the three-number set $n, j, l$. This leads to a form of radial equation for single-electron orbitals which also explicitly depends on $\alpha$ :

$$
\begin{gathered}
\frac{d f_{v}}{d r}+\frac{\kappa_{v}}{r} f_{v}(r)-\left[2+\alpha^{2}\left(\epsilon_{v}-\hat{V}_{H F}\right)\right] g_{v}(r)=0 \\
\frac{d g_{v}}{d r}-\frac{\kappa_{v}}{r} f_{v}(r)+\left(\epsilon_{v}-\hat{V}_{H F}\right) f_{v}(r)=0
\end{gathered}
$$

here $\kappa=(-1)^{l+j+1 / 2}(j+1 / 2)$, and $\hat{V}_{H F}$ is the HartreeFock potential. Equation (5) with $\alpha=\alpha_{0} \sqrt{\delta+1}$ is used to construct a full set of single-electron orbitals.

As a first step, equation (5) is used to calculate selfconsistently single-electron states of the closed-shell core. Then, this equation is used to calculate a complete set of B-spline single-electron basis orbitals. We use $40 \mathrm{~B}-$ splines of order $k=9$ in a cavity of radius $40 a_{B}$ with angular momentum $l$ up to $l_{\max }=5$. More details on the use of B-splines in atomic calculations can be found in Ref. [23].

This basis set is used to perform calculations with the linearized couple-cluster single-double method supplemented by third-order many-body perturbation theory. The all-order single-double (SD) method was discussed before in Refs. 24, 25, 26, 27, 28] and thirdorder many-body perturbation theory was discussed in
Refs. [26, 29]. We also include results from second-order MBPT to uncover the role of higher-order correlations and to estimate the numerical uncertainty due to correlations.

The SD equations are written for the coefficients of the expansion of the many-electron wave function in terms of single and double excitations from the reference HartreeFock wave function. They contain Coulomb integrals between single-electron basis orbitals as parameters. The equations are first solved iteratively for the atomic core. When convergence is achieved, a similar procedure for valence states of interest is performed.

Many-body perturbation theory is used to calculate the third-order diagrams missed in the SD method (E3 extra). Therefore, the present calculations are complete through third order while selected classes of higherorder diagrams are included in all orders. A number of earlier calculations (see, e.g. [24, 25, 26, 27, 28, 29]) prove that this approach gives very good accuracy for a wide range of monovalent atoms and ions.

The SD equations depend on the fine-structure constant only implicitly, via the values of the Coulomb integrals, which are affected by the change of the singleelectron basis states (4) due to change of $\alpha$ in the Hamiltonian (5). In other words, changes in $\alpha$ lead to changes in the basis orbitals, while the SD equations remain unchanged. The same is true for terms in the MBPT expansion. However, the change of basis means that all $\mathrm{SD}$ and E3 calculations must be redone. The E3 calculations must be repeated from scratch, but the SD iterations can be restarted from previous calculations for a different value of $\alpha$. Since the change of $\alpha$ is small, [see Eq. (2)] only a few iterations are needed to solve the SD equations for new value of $\alpha$. This significantly speeds up the calculations.

Although the method used in present work has been used many times before, the set of computer codes used in present work is new and independent of previous versions. The main reason for developing new codes was the need to have them in a form that allows easy modifications, as e.g. the change of the fine-structure constant in this calculations, or inserting extra operators (Breit interaction, specific mass shift operator, etc.) for future projects. Some features of the present realization of the methods are presented in the appendix. For third-order MBPT calculations, we apply a universal algorithm that uses a numerical description of the E3 MBPT diagrams and the same piece of computer code to calculate all of them. This algorithm will be described in detail elsewhere [30].

\section{RESULTS}

Results of calculations of energy levels of C IV, Na I, $\mathrm{Mg}$ II, Al III, Si IV, Ca II and Zn II are presented in Table I. Removal energies of the lowest $s$ and $p$ states, which are important for the analysis of quasar 
TABLE I: Removal energies of the lowest $s$ and $p$ states of $\mathrm{C} \mathrm{IV}$, Na I, Mg II, Al III, Si IV, Ca II and Zn II in different approximations, comparison with experiment $\left(\mathrm{cm}^{-1}\right)$.

\begin{tabular}{|c|c|c|c|c|c|c|c|c|c|c|}
\hline Atom & $Z$ & State & RHF & $\mathrm{E} 2$ & $\Delta^{a}$ & SD & $\Delta^{a}$ & $\mathrm{SD}+\mathrm{E} 3$ & $\Delta^{a}$ & $\operatorname{Expt}^{b}$ \\
\hline \multirow[t]{3}{*}{ C IV } & 6 & $2 s$ & 519255 & 520189 & 11 & 520233 & 55 & 520231 & 53 & 520178 \\
\hline & & $2 p_{1 / 2}$ & 454054 & 455640 & -54 & 455732 & 38 & 455727 & 33 & 455694 \\
\hline & & $2 p_{3 / 2}$ & 453927 & 455509 & -78 & 455600 & 13 & 455595 & 8 & 455587 \\
\hline \multirow[t]{3}{*}{$\mathrm{Na} \mathrm{I}$} & 11 & $3 s$ & 39952 & 41229 & -220 & 41437 & -12 & 41450 & 1 & 41449 \\
\hline & & $3 p_{1 / 2}$ & 24030 & 24412 & -81 & 24486 & -7 & 24489 & -4 & 24493 \\
\hline & & $3 p_{3 / 2}$ & 24014 & 24394 & -82 & 24468 & -8 & 24471 & -5 & 24476 \\
\hline \multirow[t]{3}{*}{ Mg II } & 12 & $3 s$ & 118824 & 121076 & -192 & 121278 & 10 & 121273 & 5 & 121268 \\
\hline & & $3 p_{1 / 2}$ & 84294 & 85453 & -145 & 85585 & -13 & 85586 & -12 & 85598 \\
\hline & & $3 p_{3 / 2}$ & 84204 & 85357 & -150 & 85488 & -19 & 85489 & -18 & 85507 \\
\hline \multirow[t]{3}{*}{ Al III } & 13 & $3 s$ & 226396 & 229319 & -127 & 229489 & 43 & 229464 & 18 & 229446 \\
\hline & & $3 p_{1 / 2}$ & 173687 & 175608 & -155 & 175751 & -12 & 175741 & -22 & 175763 \\
\hline & & $3 p_{3 / 2}$ & 173452 & 175361 & -168 & 175504 & -25 & 175494 & -35 & 175529 \\
\hline \multirow[t]{3}{*}{ Si IV } & 14 & $3 s$ & 360614 & 364033 & -60 & 364172 & 79 & 364132 & 39 & 364093 \\
\hline & & $3 p_{1 / 2}$ & 290074 & 292667 & -139 & 292802 & -4 & 292780 & -26 & 292806 \\
\hline & & $3 p_{3 / 2}$ & 289606 & 292183 & -161 & 292317 & -27 & 292296 & -48 & 292344 \\
\hline \multirow[t]{3}{*}{ Ca II } & 20 & $4 s$ & 91440 & 96173 & 425 & 96097 & 349 & 95577 & -171 & 95748 \\
\hline & & $4 p_{1 / 2}$ & 68037 & 70680 & 123 & 70761 & 204 & 70491 & -66 & 70557 \\
\hline & & $4 p_{3 / 2}$ & 67837 & 70449 & 115 & 70529 & 195 & 70262 & -72 & 70334 \\
\hline \multirow[t]{3}{*}{ Zn II } & 30 & $4 s$ & 135134 & 143835 & -1055 & 144618 & -272 & 145334 & 444 & 144890 \\
\hline & & $4 p_{1 / 2}$ & 90524 & 95249 & -1161 & 96184 & -226 & 96613 & 203 & 96410 \\
\hline & & $4 p_{3 / 2}$ & 89787 & 94372 & -1164 & 95311 & -225 & 95728 & 192 & 95536 \\
\hline
\end{tabular}

${ }^{a} \Delta=E_{\text {calc }}-E_{\text {expt }}$

${ }^{b}$ NIST, Ref. [31]

absorption data, are presented in different approximations. These include the relativistic Hartree-Fock approximation (RHF), second-order many body perturbation theory (E2), single-double (SD) approximation, and SD supplemented by third-order many-body perturbation theory $(\mathrm{SD}+\mathrm{E} 3)$. For each approximation the difference between theoretical and experimental energies is presented in the columns headed $\Delta$.

The Mg II, Al III, and Si IV ions represent an isoelectronic sequence of sodium. This sequence was considered in detail in Ref. 26]. The results of present work are in good agreement with previous calculations. Some small difference can be attributed to the difference in numerical procedures and numerical parameters (such as the number of splines, the cavity radius, maximum angular momentum, etc.)

The results in Table \show that correlations are large and are strongly dominated by second-order MBPT. However, inclusion of higher-order correlations is important and leads to a significant reduction in the differences between theoretical and experimental energies.

Table II] presents relativistic energy shifts ( $q$ coefficients) in the same approximations as the energies in Table I. One can see that the role of correlations is much less important for the $q$-coefficients than for energies. While second-order correlations give some rather small contribution to $q$, contributions from higher-order correlations are practically negligible in most cases. Only for Zn II the higher-order contributions are significantly larger than the uncertainty of the calculations. Thus,
TABLE II: Relativistic energy shifts ( $q$-coefficients, see Eq. (1) for the lowest $s$ and $p$ states of C IV, Na I, Mg II, Al III, Si IV, Ca II and Zn II in different approximations $\left(\mathrm{cm}^{-1}\right)$.

\begin{tabular}{lclrrrr}
\hline \hline Atom & $Z$ & State & RHF & \multicolumn{1}{c}{ E2 } & \multicolumn{1}{c}{ SD } & SD+E3 \\
\hline C IV & 6 & $2 s$ & 243 & 244 & 244 & 244 \\
& & $2 p_{1 / 2}$ & 139 & 141 & 142 & 142 \\
& & $2 p_{3 / 2}$ & 11 & 10 & 10 & 10 \\
Na I & 11 & $3 s$ & 51 & 57 & 59 & 59 \\
& & $3 p_{1 / 2}$ & 12 & 13 & 13 & 13 \\
& & $3 p_{3 / 2}$ & -5 & -5 & -6 & -5 \\
Mg II & \multirow{2}{*}{12} & $3 s$ & 181 & 193 & 194 & 194 \\
& & $3 p_{1 / 2}$ & 70 & 74 & 74 & 74 \\
& & $3 p_{3 / 2}$ & -20 & -23 & -23 & -23 \\
Al III & \multirow{2}{*}{13} & $3 s$ & 405 & 421 & 422 & 422 \\
& & $3 p_{1 / 2}$ & 197 & 203 & 203 & 203 \\
& & $3 p_{3 / 2}$ & -39 & -45 & -45 & -44 \\
Si IV & \multirow{2}{*}{14} & $3 s$ & 753 & 773 & 774 & 774 \\
& & $3 p_{1 / 2}$ & 416 & 425 & 425 & 425 \\
& & $3 p_{3 / 2}$ & -54 & -62 & -62 & -61 \\
Ca II & \multirow{2}{*}{20} & $4 s$ & 354 & 396 & 394 & 392 \\
& & $4 p_{1 / 2}$ & 161 & 176 & 176 & 175 \\
& & $4 p_{3 / 2}$ & -41 & -58 & -59 & -56 \\
Zn II & \multirow{2}{*}{30} & $4 s$ & 2352 & 2863 & 2873 & 2910 \\
& & $4 p_{1 / 2}$ & 990 & 1271 & 1333 & 1359 \\
& & $4 p_{3 / 2}$ & 224 & 354 & 412 & 427 \\
\hline \hline
\end{tabular}

calculations of the $q$-coefficients are more stable than the calculations of energies and, consequently, more accurate.

Breit corrections to the frequencies of the transitions 
TABLE III: Contributions to the relativistic energy shifts $(q-$ coefficients, see Eq. (11) for the $s-p$ transitions in C IV, Na I, $\mathrm{Mg}$ II, Al III, Si IV, Ca II and Zn II $\left(\mathrm{cm}^{-1}\right)$

\begin{tabular}{lcrrrr}
\hline \hline Atom & $Z$ & Transition & Dirac $^{a}$ & Breit $^{b}$ & Total \\
\hline C IV & 6 & $2 s-2 p_{1 / 2}$ & 102 & 13 & 115 \\
& & $2 s-2 p_{3 / 2}$ & 234 & -12 & 222 \\
Na I & \multirow{2}{*}{11} & $3 s-3 p_{1 / 2}$ & 46 & -1 & 45 \\
& & $3 s-3 p_{3 / 2}$ & 64 & -2 & 62 \\
Mg II & \multirow{2}{*}{12} & $3 s-3 p_{1 / 2}$ & 120 & 1 & 121 \\
& & $3 s-3 p_{3 / 2}$ & 217 & -5 & 212 \\
Al III & \multirow{2}{*}{13} & $3 s-3 p_{1 / 2}$ & 219 & 5 & 224 \\
& & $3 s-3 p_{3 / 2}$ & 467 & -9 & 458 \\
Si IV & \multirow{2}{*}{14} & $3 s-3 p_{1 / 2}$ & 348 & 13 & 361 \\
& & $3 s-3 p_{3 / 2}$ & 835 & -12 & 823 \\
Ca II & \multirow{2}{*}{20} & $4 s-4 p_{1 / 2}$ & 218 & 3 & 222 \\
& & $4 s-4 p_{3 / 2}$ & 450 & -4 & 446 \\
Zn II & \multirow{2}{*}{30} & $4 s-4 p_{1 / 2}$ & 1546 & -5 & 1541 \\
& & $4 s-4 p_{3 / 2}$ & 2472 & -20 & 2452 \\
\hline \hline
\end{tabular}

${ }^{a}$ This work, see Table

${ }^{b}$ Savukov and Dzuba, Ref. 22]

considered in present paper were calculated in Ref. [22]. These corrections are larger than the uncertainty of the $q$-coefficients from omitted higher-order correlations. Therefore, they must be included for accurate results. Table III] summarizes all significant contributions. The Dirac contributions in this Table are based on averaging the SD and SD+E3 approximations from Table Breit corrections are taken from Ref. [22].

Our final results are presented in Table IV] An estimate of the numerical uncertainty is also given and results are compared with previous calculations. There are two sources of numerical uncertainty. One is omission of certain higher-order correlations and numerical accuracy of the SD and SD+E3 calculations. This uncertainty was estimated by comparing the results in the SD and $\mathrm{SD}+\mathrm{E} 3$ approximations. Another source of uncertainty is the accuracy of calculation of Breit contribution. Breit contributions are calculated very accurately within the relativistic Hartree-Fock approximation (see Ref. 22] for details). The only uncertainty which may come from the Breit interaction is due to the fact that correlation corrections to the Breit interaction are ignored. However, these contributions are small for the relatively light atoms considered in present paper. A strong argument that the Breit contribution is calculated sufficiently accurately was presented in Ref. [22], where it was demonstrated that the inclusion of the Breit interaction brings the theoretical fine-structure intervals into perfect agreement with experiment. We use a rather conservative estimate of $20 \%$ for the accuracy of the calculations of Breit contributions.

Table IV also presents results from previous calculations. All previous calculations were done in second-order of MBPT. The work of Ref. [22] also included Breit corrections. Results of the present paper are in excellent agreement with this work. Some difference for Zn II is
TABLE IV: Relativistic energy shifts ( $q$-coefficients, see Eq. (1) for the $s-p$ transitions in C IV, Na I, Mg II, Al III, Si IV, Ca II and Zn II $\left(\mathrm{cm}^{-1}\right)$; comparison with other calculations.

\begin{tabular}{lccrrr}
\hline \hline Atom & $Z$ & Transition & \multicolumn{2}{c}{ This } & \multicolumn{2}{c}{ Other } \\
& & & \multicolumn{1}{c}{ work } & Savukov $^{a}$ & Berengut $^{b}$ \\
\hline \hline C IV & 6 & $2 s-2 p_{1 / 2}$ & $115(2)$ & 115 & $104(20)$ \\
& & $2 s-2 p_{3 / 2}$ & $222(2)$ & 221 & $232(20)$ \\
Na I & \multirow{2}{*}{11} & $3 s-3 p_{1 / 2}$ & $45(0)$ & 44 & $45(4)$ \\
& & $3 s-3 p_{3 / 2}$ & $62(0)$ & 61 & $63(4)$ \\
Mg II & \multirow{2}{*}{12} & $3 s-3 p_{1 / 2}$ & $121(1)$ & 120 & $120(10)$ \\
& & $3 s-3 p_{3 / 2}$ & $212(1)$ & 211 & $211(10)$ \\
Al III & \multirow{2}{*}{13} & $3 s-3 p_{1 / 2}$ & $224(1)$ & 223 & $216(14)$ \\
& & $3 s-3 p_{3 / 2}$ & $458(2)$ & 457 & $464(30)$ \\
Si IV & \multirow{2}{*}{14} & $3 s-3 p_{1 / 2}$ & $361(2)$ & 360 & 346 \\
& & $3 s-3 p_{3 / 2}$ & $823(2)$ & 823 & 862 \\
Ca II & \multirow{2}{*}{20} & $4 s-4 p_{1 / 2}$ & $222(1)$ & 222 & 224 \\
& & $4 s-4 p_{3 / 2}$ & $446(3)$ & 450 & 452 \\
Zn II & \multirow{2}{*}{30} & $4 s-4 p_{1 / 2}$ & $1541(7)$ & 1585 & $1584(25)$ \\
& & $4 s-4 p_{3 / 2}$ & $2452(13)$ & 2488 & $2479(25)$ \\
\hline \hline
\end{tabular}

${ }^{a}$ Second-order+Breit, Ref. [22]

${ }^{a}$ Second-order, compilation of previous results presented in Ref. [20]

due to higher-order correlations. The results are also in good agreement with early calculations compiled together in Ref. [20]. The principal sources of the small differences between the present results and those of Ref. 20] are Breit and higher-order correlation corrections.

\section{CONCLUSIONS}

In the present work, we studied the role of higherorder correlations on relativistic energy shifts of atomic frequencies used in the search for variations of the finestructure constant in quasar absorption spectra. We have demonstrated that the higher-order correlations are important for energies, bringing theoretical values into better agreement with experiment. However, higher-order correlations give very small contributions to the relativistic energy shifts ( $q$-coefficients) in all cases except Zn II. Results of the present work are in good agreement with previous calculations but are more accurate.

\section{Acknowledgments}

An important part of this work was done during V.A.D. visit to the University of Notre Dame. He would likes to thank the Department of Physics of this university for the hospitality and support. The work was partly supported by Australia Research Council. The work of W.R.J. was supported in part by NSF Grant No. PHY04-56828. 


\section{APPENDIX A: EFFICIENT WAY OF CALCULATING THE SD AND MBPT TERMS}

The coupled-cluster SD method combined with MBPT has led to an accurate description of many properties of monovalent atoms; however, it is very demanding computationally. For example, for Zn II the total number of single-electron basis states used in present calculations is 319. The total number of non-zero distinctive Coulomb integrals is about $1.7 \times 10^{8}$. All of them are used in both, the SD equations and the MBPT expansion. Calculating all Coulomb integrals in advance and keeping them in computer memory is practically impossible due to huge demand for computer memory. On the other hand, calculation of Coulomb integrals from single-electron basis functions every time they are needed makes the calculations unacceptably slow. This is even more so in the case of the relativistic energy shifts considered in present paper, since we need to run all relevant codes several times for several different values of the fine-structure constant $\alpha$.

To improve the efficiency of the codes, we use an approach in which Hartree screening functions $Y$ rather than Coulomb integrals are calculated in advance and kept in memory for efficient calculation of Coulomb integrals (a similar approach was used in Ref. [32]). The Hartree screening function $Y$ is defined as

$$
\begin{aligned}
Y_{k n m}(r)=\int \frac{r_{<}^{k}}{r_{>}^{k+1}}\left(f_{n}\left(r^{\prime}\right)\right. & f_{m}\left(r^{\prime}\right) \\
& \left.+\alpha^{2} g_{n}\left(r^{\prime}\right) g_{m}\left(r^{\prime}\right)\right) d r^{\prime}
\end{aligned}
$$

where $r_{<}=\min \left(r, r^{\prime}\right)$ and $r_{>}=\max \left(r, r^{\prime}\right)$. We also need the $\rho$ functions:

$$
\rho_{j l}(r)=f_{j}(r) f_{l}(r)+\alpha^{2} g_{j}(r) g_{l}(r)
$$

Our typical coordinate grid consists of about 1000 points. Usually all points are used to calculate $Y_{k n m}(r)$. However, there is no need to keep all points for successive calculations of the Coulomb integrals. It turns out that very little lose of accuracy is caused by using a subset of points defined as every 4 th point in the interval $1 / Z \leq r \leq R_{\text {cavity }}$, where $R_{\text {cavity }}$ is the radius of the cavity in which the $B$-splines basis orbitals are defined. By cutting off points at short distances and using only every 4 th point in between we reduce the number of points by an order of magnitude. Then, the Coulomb integrals are calculated in an extremely efficient way as

$$
q_{k}(j l m n)=\sum_{i=1}^{\mu} \rho_{j l}\left(r_{i}\right) Y_{k m n}\left(r_{i}\right) w_{i}
$$

Here $\mu \approx 100$ is number of points on the sub-grid and $w_{i}$ are weight coefficients corresponding to a particular method of numerical integration. Note that only one of two integrations for Coulomb integrals is done on a reduced sub-grid. The initial integration (A1) is done using all grid points.
[1] J-P. Uzan, Rev. Mod. Phys. 75, 403 (2003).

[2] J. K. Webb, V. V. Flambaum, C. W. Churchill, M. J. Drinkwater, and J. D. Barrow, Phys. Rev. Lett. 82, 884 (1999).

[3] V. A. Dzuba, V. V. Flambaum, and J. Webb, Phys. Rev. Lett., 82, 888 (1999).

[4] J. K. Webb, M. T. Murphy, V. V. Flambaum, V. A. Dzuba, J. D. Barrow, C. W. Churchill, J. X. Prochaska, and A. M. Wolfe, Phys. Rev. Lett. 87, 091301 (2001).

[5] M. T. Murphy, J. K. Webb, V. V. Flambaum, V. A. Dzuba, C. W. Churchill, J. X. Prochaska, J. D. Barrow, and A. M. Wolfe, Not. R. Astron. Soc. 327, 1208 (2001).

[6] M. T. Murphy, J. K. Webb, V. V. Flambaum, C. W. Churchill, and J. X. Prochaska, Not. R. Astron. Soc. 327, 1223 (2001).

[7] M. T. Murphy, J. K. Webb, V. V. Flambaum, C. W. Churchill, and J. X. Prochaska, Not. R. Astron. Soc. 327, 1237 (2001).

[8] M. T. Murphy, J. K. Webb, V. V. Flambaum, M. J. Drinkwater, F. Combes, and T. Wiklind, Not. R. Astron. Soc. 327, 1244 (2001).

[9] R. Quast, D. Reimers, and S. A. Levshakov, Astron. Astrophys. 417, L7 (2004).

[10] R. Srianad, H. Chand, P. Petitjean, and B. Aracil, As- tron. Astrophys. 417, 853 (2004); Phys. Rev. Lett. 92, 121302 (2004).

[11] R. L. Brown, and M. S. Roberts, Phys. Rev. Lett. 37, 179 (1976).

[12] L. L. Cowie and A. Songalia, Astrophys. J. 453, 596 (1995).

[13] D. A. Varshalovich, V. E. Panchuk, and A. V. Ivanchik, Astron. Lett. 22, 6 (1996).

[14] V. A. Dzuba, V. V. Flambaum, J. Webb, Phys. Rev. A59, 230 (1999).

[15] V. A. Dzuba, V. V. Flambaum, M. T. Murphy and J. K. Webb, Phys. Rev. A63, 042509 (2001).

[16] V. A. Dzuba, V. V. Flambaum, M. G. Kozlov, and M. Marchenko, Phys. Rev. A66, 022501 (2002).

[17] J. C. Berengut, V. A. Dzuba, V. V. Flambaum, and M. V. Marchenko, Phys. Rev. A70, 064101 (2004).

[18] J. C. Berengut, V. V. Flambaum, and M. G. Kozlov , Phys. Rev. A72 044501, (2005).

[19] J. C. Berengut, V. V. Flambaum, and M. G. Kozlov, Phys. Rev. A73 012504, (2006).

[20] J. C. Berengut, V. A. Dzuba, V. V. Flambaum, M. V. Marchenko, J.K. Webb, M. G. Kozlov, and M. T. Murphy, arXiv:physics/0408017 (2006).

[21] S.G. Porsev, K.V. Koshelev, I.I. Tupitsyn, M.G. 
Kozlov, D. Reimers, and S.A. Levshakov, preprint: arXiv:0708.1662 (2007).

[22] I. Savukov, and V.A. Dzuba, to be published.

[23] W. R. Johnson, S. A. Blundell, and J. Sapirstein, Phys. Rev. A37, 307 (1988).

[24] S. A. Blundell, W. R. Johnson, Z. W. Liu, and J. Sapirstein, Phys. Rev. A40, 2233 (1989).

[25] S. A. Blundell, W. R. Johnson, and J. Sapirstein, Phys. Rev. A43, 3407 (1991).

[26] M. S. Safronova, A. Derevianko, and W. R. Johnson, Phys. Rev. A58, 1016 (1998).

[27] M. S. Safronova, W. R. Johnson, and A. Derevianko, Phys. Rev. A60, 4476 (1999).

[28] U. I. Safronova and W. R. Johnson, Phys. Rev. A69, 052511 (2004).
[29] S. A. Blundell, W. R. Johnson, and J. Sapirstein, Phys. Rev. A42, 3751 (1990).

[30] V. A. Dzuba, ArXiv:physics/0710.2946 (2007).

[31] Yu. Ralchenko, F.-C. Jou, D.E. Kelleher, A. E. Kramida, A. Musgrove, J. Reader, W.L. Wiese, and K. Olsen (2007). NIST Atomic Spectra Database (version 3.1.3), [Online]. Available: http://physics.nist.gov/asd3 [2007, September 18]. National Institute of Standards and Technology, Gaithersburg, MD.

[32] V. A. Dzuba and V. V. Flambaum, Phys. Rev. A75, 052504 (2007).

[33] V. V. Flambaum and J. S. M. Ginges Phys. Rev. A72, 052115 (2005). 